\title{
Angiogenesehemmer beim platinresistenten Ovarialkarzinom
}

Patientinnen mit fortgeschrittenem Ovarialkarzinom, die auf eine platinbasierte Chemotherapie nicht ansprechen, bietet die Kombination mit einem Angiogenesehemmer eine weitere Therapieoption. Für das Ergebnis scheint der Zeitpunkt der Resistenzentwicklung gegen Platin eine Rolle zu spielen.

$\mathrm{D}$ as Ansprechen auf eine platinbasierte Chemotherapie gilt als wichtiger Prognosefaktor für Patientinnen mit rezidiviertem Ovarialkarzinom. Platinresistenz - ein Rezidiv innerhalb von 6 Monaten nach Abschluss des letzten Platinregimes - deutet auf eine schlechte Prognose hin. In der AURELIA-Studie verbesserte die Zugabe des Angiogenesehemmers Bevacizumab zur StandardMonochemotherapie signifikant das onkologische Ergebnis bei Patientinnen mit platinresistenten Ovarialkarzinom. Auf Basis der AURELIA-Daten führten die Forscher eine explorative Analyse der Therapieergebnisse abhängig vom Zeitpunkt der Resistenzentwicklung durch. Sie teilten die Patientinnen in 2
Kategorien ein: primäre Platinresistenz (PPR) bei fortschreitender Erkrankung binnen weniger als 6 Monaten nach Ende der Erstlinien-Platintherapie ( $\mathrm{n}=262)$ und sekundäre Platinresistenz (SPR) bei späterem Krankheitsprogress nach der ersten, aber innerhalb von 6 Monaten nach der Zweitlinien-Platintherapie $(\mathrm{n}=99)$.

In der Studienpopulation mit Bevacizumab als Erweiterung zur StandardMonochemotherapie ergaben sich signifikante Unterschiede im progressionsfreien Überleben (PFS): median 10,2 Monate bei SPR versus 5,5 Monate bei PPR. Dieser Vorteil zeigte sich auch im Gesamtüberleben (OS): median 22,2 versus 13,7 Monate. Die Forscher fanden hingegen keine signifikanten Unterschiede in diesen Parametern zwischen Patientinnen in der PPR- und SPRGruppe, die kein Bevacizumab erhalten hatten.

Fazit: Patientinnen mit Ovarialkarzinom, die im Verlauf eine SPR entwickeln, profitieren in höherem Maß von der Kombination Monochemotherapie plus Bevacizumab bzgl. PFS und OS als Frauen mit Platinresistenz bereits nach Erstlinientherapie. Primäre versus sekundäre Platinresistenz sollte als Stratifikationsfaktor in künftigen Studien zur Evaluierung antiangiogener Therapien des platinresistenten Ovarialkarzinoms Berücksichtigung finden.

Wolfgang Zimmermann

Trillsch F et al. Prognostic and predictive effects of primary versus secondary platinum resistance for bevacizumab treatment for platinum-resistant ovarian cancer. Ann Oncol. 2016; 27(9):1733-9.

\section{Lymphknotendissektion und Chemotherapie beim Ovarialkarzinom}

In einer großen retrospektiven Kohortenstudie wurde der Effekt der Lymphknotenentfernung im Rahmen des operativen Stagings und einer optionalen adjuvanten Chemotherapie auf das Gesamtüberleben von Patientinnen mit frühem epithelialem Ovarialkarzinom untersucht.

Ep pitheliale Ovarialkarzinome (EOC) - werden wegen des lange asymptomatischen Verlaufs meist in fortgeschrittenem Stadium diagnostiziert. Es besteht Konsens über die Wichtigkeit von Debulking-Operation und Chemotherapie in dieser Situation. Das Ausmaß des operativen Stagings in frühen Stadien, vor allem die Rolle von Lymphknotendissektion, und die adjuvante Chemotherapie bleiben umstritten. Die International Federation of Gynecology and Obstetrics (FIGO) empfiehlt die komplette paraaortale und pelvine Lymphadenektomie, ein radikaler und mit Morbidität assoziierter Eingriff. Ergebnisse einiger Studien legen ein längeres Gesamtüberleben $(\mathrm{OS})$ von Frauen mit einem frühen EOC durch eine adjuvante Chemotherapie nahe, unabhängig vom Ausmaß der Staging-Prozedur. In anderen Studien fand sich ein Nutzen der adjuvanten Chemotherapie vorwiegend für Patientinnen nach inkomplettem chirurgischem Staging.

Niederländische Forscher führten eine retrospektive Kohortenstudie zum Einfluss von Lymphknotendissektion und adjuvanter Chemotherapie auf die Lebenserwartung von Patientinnen mit frühem EOC durch. Im Netherlands Cancer Registry (NCR) fanden sich von 20002012 Daten von 3.658 Patientinnen mit den FIGO-Stadien I-IIA und IIIA1, bei $49,6 \%$ von ihnen war eine Lymphadenektomie erfolgt. Diese Patientinnen - inklusive Frauen mit Lymphknotenmetastasen
- profitierten von einem günstigeren 5-Jahres-OS gegenüber Patientinnen ohne Dissektion (89 vs. $82 \%$ ). Die Zahl entfernter Lymphknoten korrelierte mit dem OS. Für Patientinnen mit einem Stadium I-IIA mit mehr als 10 entfernten Lymphknoten verbesserte eine adjuvante Chemotherapie nicht das OS. Dies traf auch für Patientinnen mit HochrisikoKriterien zu.

Fazit: Standardmäßig sollte das operative Staging des frühen EOC die Dissektion von mindestens 10 , eventuell bis 20 Lymphknoten vorsehen, so die Forscher. Eine adjuvante Chemotherapie nach adäquater Lymphknotendissektion scheint das relative Überleben nicht zu verbessern.

Wolfgang Zimmermann

Kleppe M et al. The impact of lymph node dissection and adjuvant chemotherapy on survival: a nationwide cohort study of patients with clinical early-stage ovarian cancer. Eur J Cancer. 2016;66:83-90. 